This is a postprint version of:

Morillo, F.; Costas, R. \& Bordons, M. (2015). How is credit given to networking centres in their publications? A case study of the Spanish CIBER research structures. Scientometrics, 103(3), 923-938.

The final publication is available at Springer via http://dx.doi.org/10.1007/s11192-015-1564-z

\title{
How is credit given to networking centres in their publications? A case study of the Spanish CIBER research structures
}

\author{
Fernanda Morillo (1, 3), Rodrigo Costas (2) \& María Bordons (1) \\ (1) Centre for Human and Social Sciences (CCHS), Spanish National Research Council (CSIC), Albasanz \\ 26-28, 28037 Madrid, Spain. \\ (2) Leiden University, Centre for Science and Technology Studies (CWTS), Wassenaarseweg 62A, \\ 2333AL, Leiden, The Netherlands. \\ (3) Corresponding author. \\ E-mail addresses: fernanda.morillo@cchs.csic.es, rcostas@cwts.leidenuniv.nl, maria.bordons@cchs.csic.es
}

\section{Abstract}

The emergence of new networking research organisations is explained by the need to promote excellence in research and to facilitate the resolution of specific problems. This study focuses on a Spanish case, the Biomedical Research Networking Centres (CIBER), created through a partnership of research groups, without physical proximity, who work on common health related issues. These structures are a great challenge for bibliometricians due to their heterogeneous composition and virtual nature. Therefore, the main objective of this paper is to assess different approaches based on addresses, funding acknowledgements and authors to explore which search strategy or combination is more effective to identify CIBER publications. To this end, we downloaded all the Spanish publications from the Web of Science databases (WoS), in the subject categories of Gastroenterology/Hepatology and Psychiatry during the period 2008-2011. Our results showed that, taken alone, the dataset based on addresses identified more than $60 \%$ of all potential CIBER publications. However, the best outcome was obtained by combining it with additional datasets based on funding acknowledgements and on authors, recovering more than $80 \%$ of all possible CIBER publications without losing accuracy. In terms of bibliometric performance, all the CIBER sets showed scores above the country average, thus proving the relevance of these virtual organisations. Finally, given the increasing importance of these structures and the fact that authors do not always mention their connection to CIBER, some recommendations are offered to develop clear policies on how, when and where to specify this relationship. 
This is a postprint version of:

Morillo, F.; Costas, R. \& Bordons, M. (2015). How is credit given to networking centres in their publications? A case study of the Spanish CIBER research structures. Scientometrics, 103(3), 923-938.

The final publication is available at Springer via http://dx.doi.org/10.1007/s11192-015-1564-z

\section{Keywords}

Networking centres; Bibliometric data collection; Address analysis; Funding acknowledgements; Author analysis; Psychiatry; Gastroenterology / Hepatology.

\section{Introduction}

The need to promote excellence in research and to facilitate the solution of complex scientific problems is behind the creation of new cooperative research organisations, which benefit from specific funding strategies and management practices (Cruz-Castro, Sanz-Menéndez, \& Martínez, 2012). Networks are a special case of such organisations, since they have a very flexible structure, which enables cooperation among different research groups without physical proximity. These groups work together on common topics to achieve results with a higher relevance and to solve problems in a more efficient way (Delgado Rodríguez, 2012). Organisations of this kind can be found all across Europe, such as the European Clinical Research Infrastructure Network (ECRIN) (Demotes-Mainard \& Ohmann, 2005). In America, the Canadian Networks of Centres of Excellence serve as links between researchers from different organisations to work on common projects (Rank \& Williams, 1999). In the USA, the Research Coordination Network was created to encourage the development of cooperative research lines, surpassing disciplinary, geographical and organisational boundaries (Garner, Porter, Newman, \& Crowl, 2012).

From a bibliometric point of view, these network structures pose a major challenge due to their heterogeneous composition and virtual nature, also reflected in their scientific publications in bibliographic databases, which hinders their proper identification. For this reason, a better understanding of how these network research organisations are mentioned in scientific publications can contribute to identify their production in a more efficient and accurate way. The identification problem, which may be considered a simple technical issue, is actually an important subject, since it has a great influence on the accuracy/reliability of the results in bibliometric studies of organisations and collaboration analysis. This is reflected, for example, in the way in which specific organisations, such as academic hospitals or other institutes affiliated to universities are considered in bibliometric studies (Praal, Kosten, Calero-Medina, \& Visser, 2013). Accordingly, the proliferation of new organisational structures such as networks represents an important challenge for bibliometric research, being necessary to seek the most appropriate way to identify them. 
This is a postprint version of:

Morillo, F.; Costas, R. \& Bordons, M. (2015). How is credit given to networking centres in their publications? A case study of the Spanish CIBER research structures. Scientometrics, 103(3), 923-938.

The final publication is available at Springer via http://dx.doi.org/10.1007/s11192-015-1564-z

In this paper, we focus on a Spanish case, the Biomedical Research Networking Centres (CIBER), which were sponsored by the Spanish Consolider-Ingenio Programme ${ }^{1}$. These centres were created $^{2}$ in order to promote research excellence and translational research and to increase the critical mass in the research of specific scientific topics ${ }^{3}$. They have an origin in earlier thematic networks and their horizontal organisational model allows achieving competitive advantages by sharing objectives and resources, increasing the efficiency of results and providing a faster transfer of knowledge, hence expecting to contribute to improving the health of citizens and their quality of life. The CIBER structures were created as consortia of public and private research institutions, through partnership of research teams. These consortia have their own legal personality but without physical proximity, consisting of virtual or networked research organisations on a nationwide scale. It is also important to mention that these virtual centres have not only access to external sources of funding (e.g. Spanish or European programs), but also to internal specific programmes targeted to their members. As a result, CIBER may present certain duality because, on the one hand, they refer to structures that produce research and, on the other hand, to researchfunding schemes that provide funds to the groups included in such structures.

In a previous paper (Morillo, Díaz-Faes, González-Albo, \& Moreno, 2014), publications produced by CIBER research structures in two specific fields were identified and analysed based only on their addresses. In that study, it was pointed out that their identification was very complex, particularly given the diversity of the network participants. In fact, in this paper we will see that the CIBER structure is not always mentioned and when it does, it may appear in the address of the papers and/or in the funding acknowledgements. This latter is the case of the above-mentioned specific programmes oriented to promote collaborative research among CIBER groups. Given this scenario and the increasing importance of this type of centres, it is important to better understand how these structures can be identified in scientific publications and, particularly, to find methodologies that allow the detection of these publications in a reasonable manner.

\footnotetext{
${ }^{1}$ http://www.boe.es/boe/dias/2007/04/19/pdfs/A17366-17372.pdf. Accessed 3 Dec 2014.

2 http://www.eng.isciii.es/ISCIII/es/contenidos/fd-investigacion/fd-ejecucion/fd-centros-participados/fdconsorcios2/cibers.shtml. Accessed 3 Dec 2014.

${ }^{3}$ In 2014, CIBER conducts research in eight different research fields: bioengineering, biomaterials and nanomedicine (CIBERBBN), diabetes and metabolic disease (CIBERDEM), hepatic and digestive diseases (CIBEREHD), rare diseases (CIBERER), respiratory diseases (CIBERRES), epidemiology and public health (CIBERESP), obesity and nutrition (CIBEROBN) and mental health (CIBERSAM).
} 
This is a postprint version of:

Morillo, F.; Costas, R. \& Bordons, M. (2015). How is credit given to networking centres in their publications? A case study of the Spanish CIBER research structures. Scientometrics, 103(3), 923-938.

The final publication is available at Springer via http://dx.doi.org/10.1007/s11192-015-1564-z

\section{Objective}

This study focuses on the analysis of how CIBER research groups indicate their membership in their papers, particularly considering three main sources of metadata coming from the Web of Science (WoS) bibliographic records: authors, addresses and funding acknowledgements. Consequently, this is not a comprehensive study of CIBER performance ${ }^{4}$, but rather a methodological approach offering different possibilities to recognise CIBER publications in bibliographic databases and to explore which strategy is more efficient to identify their publications. Our concern is how to make CIBER visible in bibliometric studies without requiring specific information from the given structure. Two concrete research questions are posed:

- How do CIBER groups acknowledge their membership in scientific publications?

- Would the different forms of CIBER acknowledgement substantially affect the bibliometric measurement of its groups' performance?

Furthermore, we aim to draw some practical conclusions in order to provide advice to funding agencies and researchers (i.e. how this type of structures should be better acknowledged in order to facilitate their easier and more accurate detection).

\section{Methodology}

CIBER publications are spread over a wide number of scientific fields, but our study focuses on two WoS disciplines: Gastroenterology/Hepatology and Psychiatry. A high level of activity of CIBER structures has been previously described in these two selected fields (Morillo et al., 2014), thus ensuring we would have an appropriate number of publications to draw valid conclusions. Accordingly, all the Spanish WoS publications in Gastroenterology/Hepatology and Psychiatry during the period 2008-2011 were collected. As mentioned before, several metadata elements from the publications were extracted: addresses, funding acknowledgements and authors.

\footnotetext{
${ }^{4}$ For such type of study see, for example, Méndez Vásquez, Suñén Pinyol, Olivé Vázquez, Cervelló González, \& Camí, (2009), commissioned by the direction of the CIBERESP structure.
} 
This is a postprint version of:

Morillo, F.; Costas, R. \& Bordons, M. (2015). How is credit given to networking centres in their publications? A case study of the Spanish CIBER research structures. Scientometrics, 103(3), 923-938.

The final publication is available at Springer via http://dx.doi.org/10.1007/s11192-015-1564-z

\section{Addresses}

In the two disciplines, all publications with a CIBER affiliation or a variant form were identified based on our previous methodology (Morillo et al., 2014). For the identification of institutions, we used a previously developed application, which analyses detailed institutional data and gives optional codes to each address using the information from various master lists (Morillo, Santabárbara, \& Aparicio, 2013).

\section{Funding acknowledgements}

For all the publications in the disciplines of Gastroenterology/Hepatology and Psychiatry, we collected all their funding acknowledgements (available in the Web of Science data from August 2008 onwards, cf. Costas \& van Leeuwen, 2012). Then, in the funding agency text of the WoS records, we identified all mentions to the CIBER funding or a variant form, selecting those publications carrying one of these variants.

We decided to consider the funding acknowledgements, since we have observed that some papers only include CIBER in this section, although there are some consortia (e.g. CIBERESP, 2007) that recommend the mention of CIBER in addresses as the most important requirement.

\section{Authors}

In this step, we tried to identify authors linked to any CIBER structure. The main purpose of this approach was to allow the selection of publications that could be attributed to these structures, but did not mention CIBER in the paper, either in the address or in the funding acknowledgements metadata.

For this identification of authors, we took advantage of the list of disambiguated authors recently developed at CWTS (Caron \& van Eck, 2014). This list, apart from the connection with the entire author's output in WoS, provides the two most common affiliations of a given author in the period 1980-2012, detected through the analysis of the existing links between authors and addresses in the WoS scientific publications. Considering this list, authors with a Spanish CIBER address (or a variant form) were identified and all their publications in the two selected disciplines (Gastroenterology/Hepatology and Psychiatry) - even when CIBER was not recorded in the paper - were selected. As a result, we were able to expand the initial sets of CIBER publications (i.e. based on affiliations and acknowledgements) to other 
This is a postprint version of:

Morillo, F.; Costas, R. \& Bordons, M. (2015). How is credit given to networking centres in their publications? A case study of the Spanish CIBER research structures. Scientometrics, 103(3), 923-938.

The final publication is available at Springer via http://dx.doi.org/10.1007/s11192-015-1564-z

potential CIBER publications (i.e. also based on the presence of CIBER authors). It should be mentioned that this approach of linking authors to CIBER addresses is quite conservative. It requires that, in a large period of time (1980-2012), a substantial part of the authors' publications shows a clear connection with a CIBER affiliation. Nevertheless, in order to have an estimation of the validity of this method, we selected, for each discipline, a set from those articles that contribute to expand the initial sets of CIBER publications (i.e. based on this author expansion), creating a random sample of publications, with a confidence level of $\left.95 \%{ }^{5}\right)$. These publications and their authors were manually checked in CIBER's Annual Reports and web pages. The results showed that around $97-98 \%$ of the articles, in Psychiatry and Gastroenterology/Hepatology respectively, really belong to a CIBER structure, thus suggesting a reasonable validity for this approach.

Given the previous, it is still important to keep in mind that this author approach could however introduce a bias towards younger researchers who have started their careers linked to a CIBER structure, being less effective for senior researchers who have most of their publications linked to other organisations. For this reason, another set was created in the selected disciplines, years 2008-2011. This second set was made by the publications produced by researchers who frequently appear in papers with a CIBER affiliation, but do not necessarily have a direct linkage with that affiliation. To increase the likelihood of retrieving relevant authors we established a threshold of 10 or more publications, far enough demanding since the average number of publications of CIBER authors is less than three in papers with a CIBER affiliation. In this set, exhaustiveness was more important than precision, allowing us to expand the selection of publications. Again, to estimate the validity of this second method, we selected, for each discipline, a set from those articles that expand the other sets (i.e. only retrieved through this method), creating a new random sample of publications, with a confidence level of $95 \%{ }^{6}$ ). The results showed that, in this case, around $61-63 \%$ of the articles, in Psychiatry and Gastroenterology/Hepatology respectively, really belong to a CIBER structure. Hence, we assume that in this second approach there are further false positives (i.e. publications that do not belong to CIBER authors but to their collaborators). For this reason, it was decided to use the dataset based on CIBER authors' collaborators only as a potential upper bound of publications, particularly in order to frame and discuss the coverage and impact of the other data collections.

\footnotetext{
${ }^{5}$ The samples are composed by 61 articles for Gastroenterology/Hepatology and 107 for Psychiatry.

${ }^{6}$ The samples are composed by 115 articles for Gastroenterology/Hepatology and 132 for Psychiatry.
} 
This is a postprint version of:

Morillo, F.; Costas, R. \& Bordons, M. (2015). How is credit given to networking centres in their publications? A case study of the Spanish CIBER research structures. Scientometrics, 103(3), 923-938.

The final publication is available at Springer via http://dx.doi.org/10.1007/s11192-015-1564-z

\section{CIBER sets}

In order to study the effects of the various ways of identifying CIBER publications, we have created the following sets of papers in each discipline:

- CIBER_address: all publications detected through the affiliation section (as in Morillo et al., 2014).

- CIBER_funding: all publications detected through the funding acknowledgements section.

- Author_CIBER: all publications that belong to authors directly linked to a CIBER structure.

- Author_CIBERorColl: all publications that belong to authors directly linked to a CIBER structure (Author_CIBER) or with at least 10 publications in the CIBER_address set.

In addition, for each discipline, we have created a counterpart of each CIBER set, called 'Others'. This counterpart includes all other Spanish publications that do not belong to the specific CIBER set (e.g. 'CIBER_address'). The main purpose of creating these sets is to analyse inter-sets differences in the number of publications, but also in impact. Finally, we test the combination of these sets to discuss which is the best option.

\section{Bibliometric indicators}

Some bibliometric indicators were obtained for each selected discipline, Gastroenterology/Hepatology and Psychiatry, for the different sets of CIBER papers identified through the approaches mentioned above and for their counterparts. The following data were shown to describe research performance: number of articles over the period 20082011, average number of authors and institutions, mean normalised citation score (MNCS); percentage of the top $10 \%$ highly cited papers in their disciplines (PPTop); mean normalised journal citation score (MNJS) ${ }^{7}$; percentage of collaboration (\%Coll) and percentage of international collaboration (\%IntColl). It should be noted that the percentage of collaboration

\footnotetext{
${ }^{7}$ For a discussion on these field-normalised indicators check Waltman, van Eck, van Leeuwen, Visser, \& van Raan (2011)
} 
This is a postprint version of:

Morillo, F.; Costas, R. \& Bordons, M. (2015). How is credit given to networking centres in their publications? A case study of the Spanish CIBER research structures. Scientometrics, 103(3), 923-938.

The final publication is available at Springer via http://dx.doi.org/10.1007/s11192-015-1564-z

includes any kind of collaboration, regardless its scope, national or international, interinstitutional or intra-institutional.

Differences in the number of CIBER papers of the various sets were analysed. In addition, differences in the measures of research performance, in terms of citation impact, were considered. Finally, the combination of different sets was explored to determine which was the optimum approach for a comprehensive collection of CIBER publications and to what extent the bibliometric results may change depending on the search strategy used.

\section{Results}

\section{General results}

In this section, we offer some basic results concerning the description of the publication activity of Spanish scientists in the two disciplines under study. A total of 3678 articles were included in the analysis. Figure 1, which shows the evolution of the number of articles for both disciplines, presents a positive increment in the number of Spanish publications. The growth rate in Psychiatry was $25 \%$ and $27 \%$ for Gastroenterology/Hepatology. If we take into account that the growth rate in the whole WoS database for the period $2008-2011$ is $10 \%$, we can state that this growth is not only caused by the coverage expansion of WoS.

Figure 1. Evolution of the number of Spanish articles in Gastroenterology/Hepatology and Psychiatry.

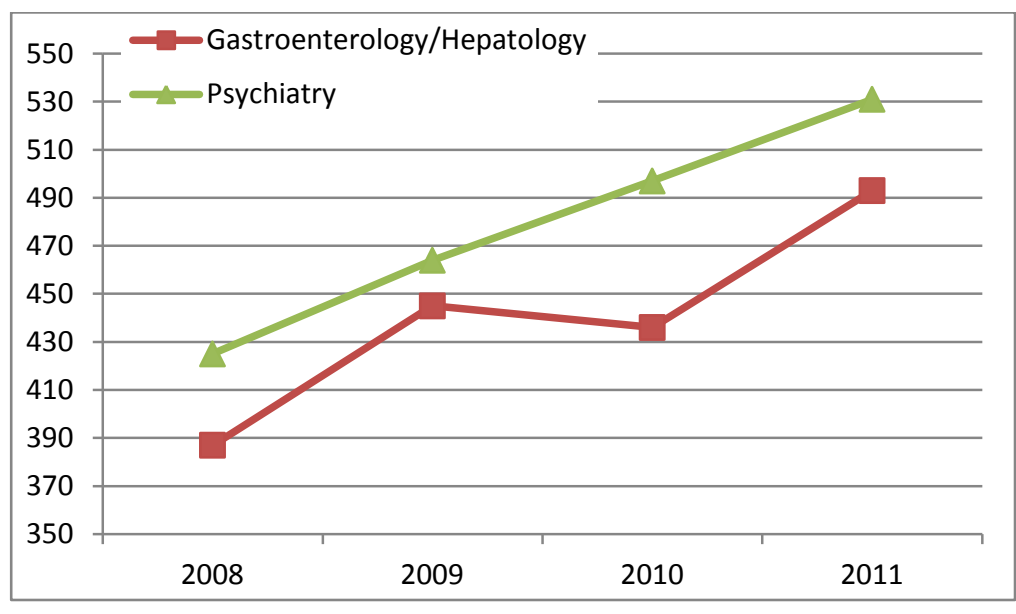

Table 1 shows the main average values for the different indicators considered in the analysis. In general, both fields show a similar pattern in the average number of authors and 
This is a postprint version of:

Morillo, F.; Costas, R. \& Bordons, M. (2015). How is credit given to networking centres in their publications? A case study of the Spanish CIBER research structures. Scientometrics, 103(3), 923-938.

The final publication is available at Springer via http://dx.doi.org/10.1007/s11192-015-1564-z

collaborative organisations per publication, although Gastroenterology/Hepatology shows a slightly higher level in the first one and a lower level in the second one than Psychiatry. In terms of the field normalised impact (MNCS and PPTop) and the journal-normalised impact (MNJS), Spanish publications in both disciplines present relatively similar performance, slightly higher for Gastroenterology/Hepatology in the field normalised impact and slightly higher for Psychiatry in the journal normalised impact. Nevertheless, collaboration is quite different between disciplines, with higher percentages for Psychiatry than for Gastroenterology/Hepatology, mainly in international collaboration.

Table 1. Spanish publications in Gastroenterology/Hepatology and Psychiatry: number of articles, average number of authors and institutions, citation data and percentages of collaboration (WoS, 2008-2011)

\begin{tabular}{|lcrrrrrrr|}
\hline \multicolumn{1}{|c}{ Discipline } & Art & AvgAuth & AvgOrg & MNCS & PPTop & MNJS & \%Coll & \%IntColl \\
\hline Gastroenterology/Hepatology & 1761 & 7.75 & 3.88 & 1.19 & 13.96 & 1.05 & 76.49 & 27.65 \\
\hline Psychiatry & 1917 & 7.67 & 4.76 & 1.14 & 11.93 & 1.08 & 85.55 & 44.18 \\
\hline
\end{tabular}

AvgAuth: average number of authors; AvgOrg: average number of organisations; MNCS: mean normalised citation score; PPTop: percentage of the top $10 \%$ highly cited papers; MNJS: mean normalised journal citation score; \%Coll: percentage of collaboration; \%IntColl: percentage of international collaboration.

\section{CIBER sets}

The numbers of papers retrieved through the different methods used for the identification of the CIBER output are shown in Table 2. The set based on CIBER authors and collaborators (Author_CIBERorColl) accounted for the highest number of papers (780 and 921 articles in Gastroenterology/Hepatology and Psychiatry, respectively), while the lowest recall corresponded to papers including CIBER in the acknowledgement field (72 and 245 in Gastroenterology/Hepatology and Psychiatry, respectively). It should be noted that some papers might be retrieved by more than one of the sets; this explains why the last row is lower than the sum, since it only includes unique papers.

Table 2. Number of CIBER papers by set and discipline.

\begin{tabular}{|lrrrr|}
\hline \multicolumn{1}{|c}{ Set } & $\begin{array}{c}\text { Gastroenterology } \\
\text { /Hepatology }\end{array}$ & \% Gastro & Psychiatry & \% Psych \\
\hline CIBER_address & 633 & 71.77 & 636 & 61.21 \\
\hline CIBER_funding & 72 & 8.16 & 245 & 23.58 \\
\hline Author_CIBER & 350 & 39.68 & 440 & 42.35 \\
\hline Author_CIBERorColl & 780 & 88.44 & 921 & 88.64 \\
\hline $\begin{array}{l}\text { ClBER_address + CIBER_funding } \\
\text { + Author_CIBERorColl }\end{array}$ & 882 & 100.00 & 1039 & 100.00 \\
\hline
\end{tabular}


This is a postprint version of:

Morillo, F.; Costas, R. \& Bordons, M. (2015). How is credit given to networking centres in their publications? A case study of the Spanish CIBER research structures. Scientometrics, 103(3), 923-938.

The final publication is available at Springer via http://dx.doi.org/10.1007/s11192-015-1564-z

The address set is the most useful single item of information for identifying CIBER structures (around $60-70 \%$ of all papers, Table 2), while Author_CIBERorColl brings the highest percentage of unique papers in both disciplines. Moreover, Figure 2 shows that common papers, retrieved by at least two different sets, represented around $60 \%$ of unique papers (553 articles in Gastroenterology/Hepatology and 600 in Psychiatry). The individual additional contribution of each set ranges from 1\% (CIBER_funding) to 18-19\% (Author_Coll, without Author_CIBER).

Figure 2. Percentages of papers gathered by the different sets.
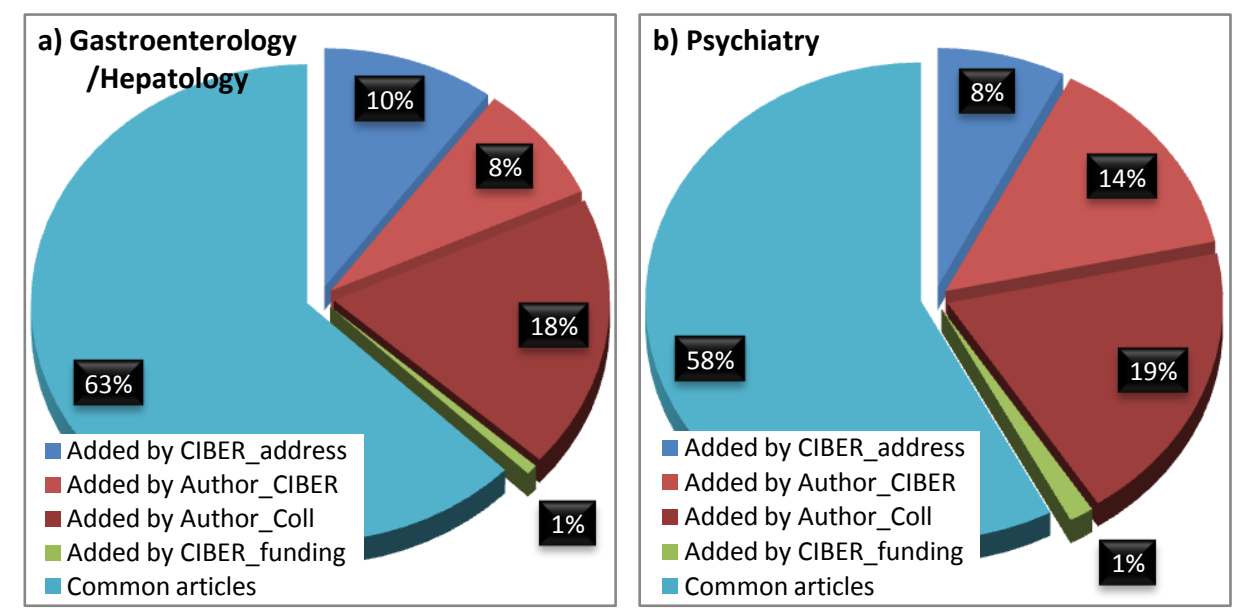

Note: common articles are those retrieved by at least two different sets. The remaining categories of articles are unique to each of the sets. ( $\mathrm{N}=882$ papers in Gastroenterology/Hepatology; $\mathrm{N}=1039$ in Psychiatry)

Table 3, for each set (column), shows the number of papers retrieved only by a single method, as well as the number of papers retrieved at the same time by two different sets and by the three of them together (row "Three sets combined" = CIBER_address + CIBER_funding + Author_CIBERorColl). Besides, the total number of articles for each set is also shown (row "Total set"), obtained by summing all of the items of each column ${ }^{8}$. On the one hand, around $36-55 \%$ of the potential papers were retrieved by both the CIBER_address and the Author_CIBERorColl sets in the two disciplines. On the other hand, the greatest single contribution can be found in the Author_CIBERorColl sets.

\footnotetext{
${ }^{8}$ e.g. for Gastroenterology/Hepatology, the CIBER_address set includes articles retrieved through the address field, whether alone (89) or in combination with a) the funding field (7), b) CIBER authors and collaborators $(487), c)$ these two sets together (50). All these numbers sum 633 articles.
} 
This is a postprint version of:

Morillo, F.; Costas, R. \& Bordons, M. (2015). How is credit given to networking centres in their publications? A case study of the Spanish CIBER research structures. Scientometrics, 103(3), 923-938.

The final publication is available at Springer via http://dx.doi.org/10.1007/s11192-015-1564-z

Table 3. Combinations of the three major methods by discipline.

\begin{tabular}{|lrrr|}
\hline $\begin{array}{l}\text { Gastroenterology } \\
\text { /Hepatology }\end{array}$ & CIBER_address & CIBER_funding & Author_CIBERorColl \\
\hline CIBER_address & 89 & 7 & 487 \\
\hline CIBER_funding & 7 & 6 & 9 \\
\hline Author_CIBERorColl & 487 & 9 & 234 \\
\hline Three sets combined & 50 & 50 & 50 \\
\hline Total set & 633 & 72 & 780 \\
\hline \multicolumn{1}{|c|}{ Psychiatry } & CIBER_address & CIBER_funding & Author_CIBERorColl \\
\hline CIBER_address & 78 & 25 & 370 \\
\hline CIBER_funding & 25 & 15 & 42 \\
\hline Author_CIBERorColl & 370 & 42 & 346 \\
\hline Three sets combined & 163 & 163 & 163 \\
\hline Total set & 636 & 245 & 921 \\
\hline
\end{tabular}

The annual evolution of publications retrieved by each of the methodologies is shown in Figure 3 for the two fields analysed $(a, b)$. As we can see, a positive increase in the number of papers retrieved over time is observed for all the sets. We can also see how the set of Author_CIBERorColl was the one that produced more publications in all years, followed by CIBER_address, Author_CIBER and CIBER_funding. Nevertheless, CIBER_funding was the set with the greatest growth, followed by the CIBER_address, Author_CIBER and Author_CIBERorColl ones. Furthermore, in the case of Psychiatry, the first two sets showed a significant rise, which can also be seen in the figure, mainly in the case of the CIBER_address set. This may be explained because the Mental Health CIBER (CIBERSAM), although representing on average $80 \%$ of the CIBER papers of Psychiatry, in 2008 it only contributes about $60 \%$ of them. That is, in 2008 , only a few CIBERSAM authors sign with their CIBER affiliation, which can be due to the recent creation of this consortium at the end of 2007. 
This is a postprint version of:

Morillo, F.; Costas, R. \& Bordons, M. (2015). How is credit given to networking centres in their publications? A case study of the Spanish CIBER research structures. Scientometrics, 103(3), 923-938.

The final publication is available at Springer via http://dx.doi.org/10.1007/s11192-015-1564-z

Figure 3. Evolution of the number of articles retrieved in the different sets

a) Gastroenterology/Hepatology

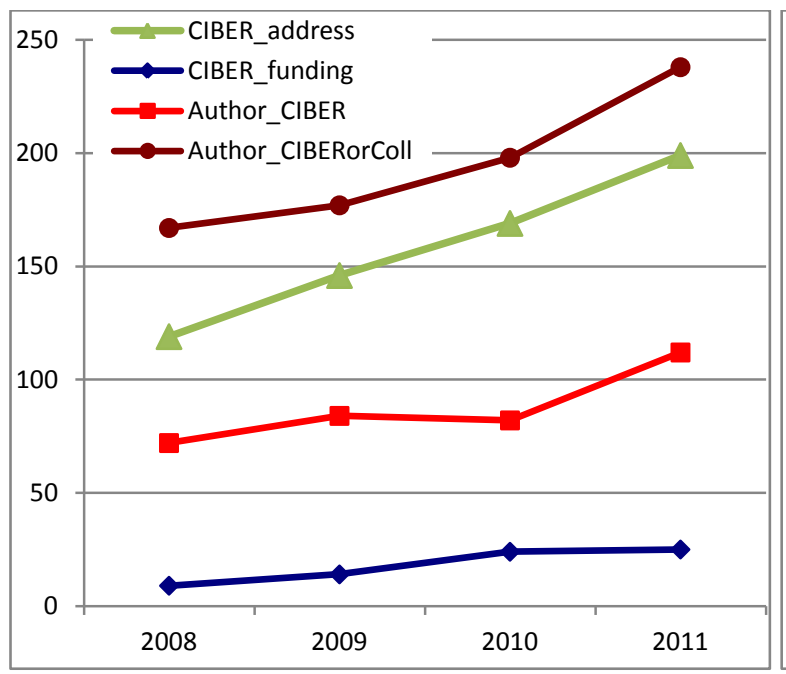

b) Psychiatry

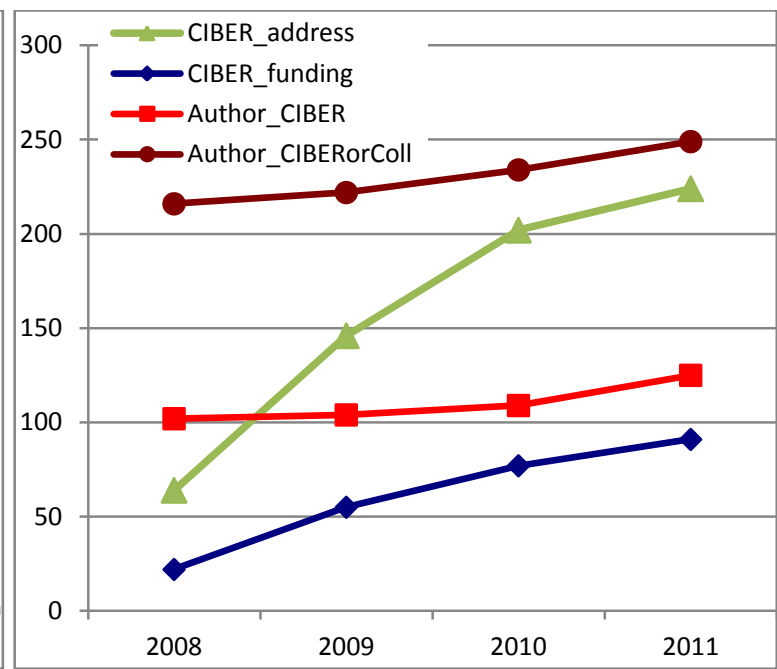

Data regarding activity, impact and collaboration for the different datasets in Gastroenterology/Hepatology are shown in Table 4. The original set, the one with CIBER addresses, offered the highest results in the percentage of international collaboration (\%IntColl). On the other side, the set Author_CIBER stood out in two important variables, the percentage of highly cited papers (PPTop) and the percentage of collaboration (\%Coll), while having the greatest number of average authors and organisations. Finally, the set with collaborators (Author_CIBERorColl) highlighted in mean paper citations (MNCS) and in the total number of articles.

Table 4. Research performance for the different sets of CIBER papers in Gastroenterology/Hepatology

\begin{tabular}{|crrrrrrrrr|}
\hline \multicolumn{1}{c}{ Set } & \multicolumn{1}{c}{ Art } & AvgAuth & AvgOrg & MNCS & PPTop & MNJS & \%Coll & \%IntColl \\
\hline CIBER_address & 633 & 8.15 & 4.03 & 1.58 & 20.49 & 1.44 & 78.83 & 34.44 \\
\hline Others & 1128 & 7.53 & 3.80 & 0.96 & 10.30 & 0.83 & 75.18 & 23.85 \\
\hline CIBER_funding & 72 & 8.29 & 3.15 & 1.45 & 14.71 & 1.48 & 75.00 & 25.00 \\
\hline Others & 1689 & 7.73 & 3.91 & 1.18 & 13.93 & 1.03 & 76.55 & 27.77 \\
\hline Author_CIBER & 350 & 9.10 & 4.36 & 1.44 & 20.49 & 1.32 & 82.57 & 26.86 \\
\hline Others & 1411 & 7.42 & 3.77 & 1.12 & 12.34 & 0.98 & 74.98 & 27.85 \\
\hline Author_CIBERorColl & 780 & 8.56 & 4.32 & 1.60 & 20.41 & 1.36 & 80.38 & 31.79 \\
\hline Others & 981 & 7.10 & 3.54 & 0.86 & 8.83 & 0.80 & 73.39 & 24.36 \\
\hline
\end{tabular}

AvgAuth: average number of authors; AvgOrg: average number of organisations; MNCS: mean normalised citation score; PPTop: percentage of the top 10\% highly cited papers; MNJS: mean normalised journal citation score; \%Coll: percentage of collaboration; \% IntColl: percentage of international collaboration. 
This is a postprint version of:

Morillo, F.; Costas, R. \& Bordons, M. (2015). How is credit given to networking centres in their publications? A case study of the Spanish CIBER research structures. Scientometrics, 103(3), 923-938.

The final publication is available at Springer via http://dx.doi.org/10.1007/s11192-015-1564-z

Table 5 presents results for Psychiatry and offers similar performance for the author sets (CIBER and collaborators). It should be noted that the CIBER_funding set outstands in every variable, although with a low number of articles. However, the original CIBER_address set offers also good results in all variables, reaching the second place even in the number of articles.

Table 5. Research performance for the different sets of CIBER papers in Psychiatry

\begin{tabular}{|lrrrrrrrrr|}
\hline \multicolumn{1}{c}{ Set } & \multicolumn{1}{c}{ Art } & AvgAuth & AvgOrg & MNCS & PPTop & MNJS & \%Coll & \%IntColl \\
\hline ClBER_address & 636 & 9.00 & 5.61 & 1.45 & 16.78 & 1.26 & 89.78 & 50.94 \\
\hline Others & 1281 & 7.01 & 4.34 & 0.98 & 9.52 & 0.99 & 83.45 & 40.83 \\
\hline ClBER_funding & 245 & 9.61 & 6.04 & 1.61 & 19.01 & 1.47 & 91.02 & 55.51 \\
\hline Others & 1672 & 7.39 & 4.57 & 1.07 & 10.89 & 1.02 & 84.75 & 42.52 \\
\hline Author_ClBER & 440 & 8.97 & 5.22 & 1.33 & 14.51 & 1.22 & 87.73 & 45.00 \\
\hline Others & 1477 & 7.29 & 4.62 & 1.08 & 11.16 & 1.04 & 84.90 & 43.94 \\
\hline Author_ClBERorColl & 921 & 8.54 & 5.06 & 1.32 & 14.57 & 1.21 & 89.25 & 45.28 \\
\hline Others & 996 & 6.87 & 4.48 & 0.96 & 9.48 & 0.96 & 82.13 & 43.17 \\
\hline
\end{tabular}

AvgAuth: average number of authors; AvgOrg: average number of organisations; MNCS: mean normalised citation score; PPTop: percentage of the top 10\% highly cited papers; MNJS: mean normalised journal citation score; \%Coll: percentage of collaboration; \% IntColl: percentage of international collaboration.

It is very interesting to remark that although the number of identified CIBER articles varies largely depending on the search strategy, in all cases the final set of papers retrieved shows better performance, in terms of impact, than the remaining papers in their corresponding disciplines.

\section{Combinations of sets}

Once we have analysed the recall and performance of the different sets separately, we wanted to explore how different could be the results, in terms of publications and impact, depending on the potential grouping of these sets. In this sense, we considered the following combinations:

1) CIBER_address + CIBER_funding: this is the simplest approach, since it can be collected directly looking for the appropriate terms in the WoS records' metadata. However, information from funding acknowledgements is still quite difficult to process (Sirtes, 2013) and it is not clear how Thomson Reuters collects and handles this information (Costas \& van Leeuwen, 2012). For example, in WoS, the acknowledgement information is systematically missing in publications produced in other languages than English (Díaz- 
This is a postprint version of:

Morillo, F.; Costas, R. \& Bordons, M. (2015). How is credit given to networking centres in their publications? A case study of the Spanish CIBER research structures. Scientometrics, 103(3), 923-938.

The final publication is available at Springer via http://dx.doi.org/10.1007/s11192-015-1564-z

Faes \& Bordons, 2014). Moreover, mistakes in the affiliation data and/or funding acknowledgements are a quite common problem in bibliometrics (García-Zorita, MartínMoreno, Lascurain-Sánchez, \& Sanz-Casado, 2006; Jiang, Zheng, Wang, Lu, \& Wu, 2011; Sirtes, 2013).

2) CIBER_address + CIBER_funding + Author_CIBER: this approach provides a higher number of publications and is also quite accurate. The reason is that Author_CIBER publications belong to authors who have a strong direct linkage with a CIBER structure (as found in the WoS publications). However, although it is a valid way for identifying additional CIBER publications, we should not underestimate the fact that it poses a relevant technical drawback, mostly because working with names is very problematic, as their disambiguation is time consuming and in many cases very difficult (Costas \& Bordons, 2007; Reijnhoudt, Costas, Noyons, Boerner, \& Scharnhorst, 2013; Smalheiser \& Torvik, 2009).

3) CIBER_address + CIBER_funding + Author_CIBERorColl: this approach provides the highest number of publications, although with less precision, as it contains noise due to authors not really affiliated to any CIBER. It is important to remind that our data collection system for Author_CIBERorColl consists of detecting authors who frequently appear in the set of CIBER_address, but this does not mean that they have a direct link with a CIBER structure ${ }^{9}$.

Notwithstanding, we can take this last combination of sets as an upper bound of potential CIBER publications given the fact that it is the most exhaustive publication set. In view of this, the second combination gathered more than $80 \%$ of all the potential publications collected in the third one, for both fields, while showing slightly higher impact values. Interestingly enough, the first combination collected around $70 \%$ of possible CIBER publications in Gastroenterology/Hepatology and Psychiatry respectively, whereas its values for impact were not very different or even better to those obtained in the other combinations. Besides, in all cases, CIBER publications detected through any combination of sets showed higher impact than the rest of the publications in the same fields (Table 6 and Table 7).

\footnotetext{
${ }^{9}$ It should be noted that authors and affiliations are not always clearly connected in WoS publications (Reijnhoudt, Costas, Noyons, Boerner, \& Scharnhorst, 2013).
} 
This is a postprint version of:

Morillo, F.; Costas, R. \& Bordons, M. (2015). How is credit given to networking centres in their publications? A case study of the Spanish CIBER research structures. Scientometrics, 103(3), 923-938.

The final publication is available at Springer via http://dx.doi.org/10.1007/s11192-015-1564-z

Table 6. Comparison of CIBER performance by combination of sets: Gastroenterology/Hepatology

\begin{tabular}{|c|c|c|c|c|c|c|c|c|}
\hline Set & Art & AvgAuth & AvgOrg & MNCS & PPTop & MNJS & \%Coll & \%IntColl \\
\hline 1) CIBER_address + CIBER_funding & 648 & 8.14 & 4.01 & 1.58 & 20.32 & 1.44 & 78.55 & 34.10 \\
\hline 1) Others & 1113 & 7.52 & 3.81 & 0.96 & 10.26 & 0.82 & 75.29 & 23.90 \\
\hline 2) CIBER_address + CIBER_funding + Author_CIBER & 719 & 8.37 & 4.17 & 1.57 & 20.59 & 1.41 & 79.00 & 32.68 \\
\hline 2) Others & 1042 & 7.32 & 3.69 & 0.92 & 9.39 & 0.80 & 74.76 & 24.18 \\
\hline 3) CIBER_address + CIBER_funding + Author_CIBERorColl & 882 & 8.40 & 4.24 & 1.56 & 19.60 & 1.35 & 79.25 & 32.65 \\
\hline 3) Others & 879 & 7.10 & 3.52 & 0.81 & 8.30 & 0.75 & 73.72 & 22.64 \\
\hline
\end{tabular}

Table 7. Comparison of CIBER performance by combination of sets: Psychiatry

\begin{tabular}{|c|c|c|c|c|c|c|c|c|}
\hline Set & Art & AvgAuth & AvgOrg & MNCS & PPTop & MNJS & $\%$ Coll & \%IntColl \\
\hline 1) CIBER_address + CIBER_funding & 693 & 8.95 & 5.57 & 1.41 & 16.09 & 1.26 & 90.33 & 51.08 \\
\hline 1) Others & 1224 & 6.95 & 4.30 & 0.98 & 9.57 & 0.98 & 82.84 & 40.28 \\
\hline 2) CIBER_address + CIBER_funding + Author_CIBER & 840 & 8.79 & 5.40 & 1.38 & 15.74 & 1.23 & 89.76 & 49.05 \\
\hline 2) Others & 1077 & 6.80 & 4.26 & 0.95 & 8.95 & 0.96 & 82.27 & 40.39 \\
\hline 3) CIBER_address + CIBER_funding + Author_CIBERorColl & 1039 & 8.54 & 5.20 & 1.32 & 14.62 & 1.21 & 89.61 & 46.49 \\
\hline 3) Others & 878 & 6.65 & 4.24 & 0.92 & 8.74 & 0.93 & 80.75 & 41.46 \\
\hline
\end{tabular}

\section{Discussion and conclusions}

As we stated before, networking research organisations can be found in different countries, since they establish flexible structures that enhance the development of efficient scientific activities. Therefore, it is relevant to pay attention and analyse these structures, because overlooking them could lead to their invisibility or to the misunderstanding of cooperation in bibliometric studies. On the one hand, it is important to detect the presence of research networks in publications. On the other hand, the placement of CIBER as a separate affiliation from the main address of the authors may imply the overestimation of collaborative publications.

Besides, it is noteworthy the difficulty in identifying their production, particularly considering the lack of standardisation of such organisations, probably because both scientists and managers are still unaware of this problem. For that reason, based on our results, we offer 
This is a postprint version of:

Morillo, F.; Costas, R. \& Bordons, M. (2015). How is credit given to networking centres in their publications? A case study of the Spanish CIBER research structures. Scientometrics, 103(3), 923-938.

The final publication is available at Springer via http://dx.doi.org/10.1007/s11192-015-1564-z

some advice on what methodologies are best suited in order to improve the bibliographic identification of these structures.

- Our study showed that, considering each set individually, the one based on addresses identified CIBER publications better than other sets. On the one hand, this could be explained by the fact that authors have developed a strong culture of mentioning their affiliations, while indicating funding acknowledgements is still a less extended practice and depends on the discipline and on the availability of funding. For example, in the case of biomedical fields, a high rate of funding acknowledgements has been described (Costas \& van Leeuwen, 2012), particularly for Spanish authors (Díaz-Faes \& Bordons, 2014). On the other hand, it should be borne in mind that some CIBER structures recommend their authors to mention them in addresses (CIBERESP, 2007). Nevertheless, we cannot ignore the fact that if we consider only addresses, around 30$40 \%$ of other possible CIBER publications (e.g. in Psychiatry) could be lost.

- In addition, the CIBER_address + CIBER_funding combination of sets brought more publications than the CIBER_address set alone and presented quite robust indicators in impact (even higher when compared to all potential CIBER publications, i.e. the combination of CIBER_address + CIBER_funding + Author_CIBERorColl). Besides, it was a considerable cost efficient approach.

- In general, the best approach seems to be the combination of CIBER_address + CIBER_funding + Author_CIBER sets, because we got around $80 \%$ of all possible CIBER publications, obtaining a fairly reasonable accuracy. Moreover, impact values remained higher as compared to all the potential CIBER publications detected through the combination of CIBER_address + CIBER_funding + Author_CIBERorColl.

Furthermore, we confirmed that with any of the methodologies used above, higher impact indicators were always obtained for the CIBER sets as compared to the rest of the Spanish publications in the same field. Accordingly, it can be concluded that in general CIBER outcomes are above the Spanish average and that they are fulfilling their mission of conducting excellent research. We should also mention, that this higher impact of CIBER outcomes are somehow in line with the results obtained by Costas and van Leeuwen (2012) and Wang and Shapira (2014), who detected that publications with funding support tend to have higher impact as compared to those without funding. However, in the particular case of CIBER, different factors converge to explain its higher impact, such as: a) the fact that teams 
This is a postprint version of:

Morillo, F.; Costas, R. \& Bordons, M. (2015). How is credit given to networking centres in their publications? A case study of the Spanish CIBER research structures. Scientometrics, 103(3), 923-938.

The final publication is available at Springer via http://dx.doi.org/10.1007/s11192-015-1564-z

need to pass an evaluation to be able to form part of a CIBER structure, which guarantees the high profile of most of its members. b) There is specific funding for CIBER teams, which enhances their activity ${ }^{10}$. c) There are technological platforms and additional resources within the networks, which provide highly specialised services to their members.

Finally, we could state that authors did not always mention their connection to a CIBER structure. For example, even when considering only the Author_CIBER set (i.e. a quite accurate approach for detecting authors with a clear CIBER link), we could see that around $20-30 \%$ of their publications still missed some explicit mention to the CIBER research structure. Although this could be caused by recent changes in researchers' affiliations (e.g. entering or leaving the CIBER structure and so having production elsewhere), it is very likely that some publications could still be reasonably related to a CIBER structure. In fact, Delgado Rodriguez (2012) comments that, although it may seem unusual, it is difficult to know the exact number of researchers linked to one of these structures. For this reason, it is critical to develop clear policies on how authors should indicate their relationship with these research structures and when and where should they indicate them.

\section{Final recommendations}

Based on the results and main outcomes of this research, we suggest below some basic recommendations for science policy makers and researchers, in order to enhance the future identification of CIBER and networked structures in scientific publications.

When to mention CIBER affiliations and funding? It would be important to specify in a clear way in which cases the authors must include their CIBER linkage as an affiliation and in which other cases as a funding acknowledgement, or both. In the public official documentation about CIBER organisations, it is stated that scientific publications have to mention whether they are the result of the activities carried out at a CIBER consortia, but they do not detail which are these activities and how indviduals must state their CIBER support. As an example, some consortia, as CIBERESP (2007), indicate that this should be mentioned in the researchers' affiliations. However, this organisation also mentions that this recognition may "exceptionally be required in the acknowledgements section". Nevertheless, there is no information on what are these "exceptional" circumstances when the CIBER support should be mentioned in the acknowledgements section; hence, guidelines that are more detailed would be advisable. This ambiguity can cause doubts among the authors on

\footnotetext{
${ }^{10}$ http://www.ciberisciii.es/media/5281/estatutos ciber boe-a-2014-2026.pdf. Accessed 3 Dec 2014.
} 
This is a postprint version of:

Morillo, F.; Costas, R. \& Bordons, M. (2015). How is credit given to networking centres in their publications? A case study of the Spanish CIBER research structures. Scientometrics, 103(3), 923-938.

The final publication is available at Springer via http://dx.doi.org/10.1007/s11192-015-1564-z

when and where they have to mention their membership, thus creating inconsistencies in the statement of their affiliations.

How to mention CIBER membership or support? The development of standard and clear affiliation policies for these organisations is essential in order to improve the future identification and data collection of their results. In fact, among research funders, there are already policies recommending specific forms of recognition of grant support (e.g. the US $\mathrm{NIH}^{11}$ ). Again, as an example, CIBERESP (2007) recommends a particular text ("CIBER Epidemiología y Salud Pública (CIBERESP), Spain") for both the affiliation and acknowledgements section. Indeed this is an important practice that should be spread to all CIBER consortia. Among other elements, the name of the specific network involved in the research could be included, and not only the general term CIBER (for example, CIBERESP stands for Biomedical Research Centre Network for Epidemiology and Public Health), keeping in mind that very long, complex and too detailed affiliations might discourage researchers' compliance.

In conclusion, these recommendations may seem obvious and actually, they would be applicable to any research organisation or research-funding agency. Nevertheless, we can argue that our recommendations are more relevant for network and virtual organisations such as CIBER structures, because their lack of a physical entity can seriously challenge their study and future evaluation and sustainability. Consequently, to increase the visibility and recognition of the outcomes of their activities, it is very important to find a straightforward identification of these organisations. Clearly, future research should focus on a better understanding of these organisations and the way they are mentioned and credited in scientific publications by the scholars supported by them. The results in this paper somehow suggest that in a near future we could face completely new research structures where the physical location of scientists, their sources of funding or the development of their activities become virtual and networked, thus posing new challenges for bibliometric and research policy studies.

\footnotetext{
${ }^{11}$ http://grants.nih.gov/grants/acknow.htm. Accessed 3 Dec 2014.
} 
This is a postprint version of:

Morillo, F.; Costas, R. \& Bordons, M. (2015). How is credit given to networking centres in their publications? A case study of the Spanish CIBER research structures. Scientometrics, 103(3), 923-938.

The final publication is available at Springer via http://dx.doi.org/10.1007/s11192-015-1564-z

\section{Acknowledgements}

This work is supported by the Spanish Ministry of Science and Innovation (Grant CSO2011-25102). We would like to thank the anonymous reviewer for the valuable and constructive comments on this paper.

\section{References}

Caron, E., \& van Eck, N. J. (2014). Large scale author name disambiguation using rule-based scoring and clustering. In Proceedings of STI 2014 Leiden. Retrieved from http://sti2014.cwts.nl/download/f-y2w2.pdf. Accessed 3 Dec 2014.

CIBERESP (2007, July 19). FIRMA CIBERESP. Retrieved from http://www.ciberesp.es/files/Firma CIBERESP.pdf. Accessed 3 Dec 2014.

Costas, R., \& Bordons, M. (2007). Algoritmos para solventar la falta de normalización de nombres de autor en los estudios bibliométricos._Investigación Bibliotecológica, 21(42), 13-32.

Costas, R., \& van Leeuwen, T. N. (2012). Approaching the 'reward triangle': General analysis of the presence of funding acknowledgments and 'peer interactive communication' in scientific publications. Journal of the American Society for Information Science and Technology, 63(8), 16471661. doi:10.1002/asi.22692

Cruz-Castro, L., Sanz-Menéndez, L., \& Martínez, C. (2012). Research centers in transition: patterns of converge and diversity. Journal of Technology Transfer, 37(1), 18-42. doi:10.1007/s10961-0109168-5.

Delgado Rodríguez, M. (2012). CIBERESP: Un instrumento para la promoción y la difusión de la investigación de excelencia española. Gaceta Sanitaria, 26(5), 393-394.

doi:10.1016/i.gaceta.2012.05.008

Demotes-Mainard, J., \& Ohmann, C. (2005). European clinical research infrastructures network: Promoting harmonisation and quality in European clinical research. The Lancet, 365(9454), 107108. doi:10.1016/S0140-6736(05)17720-4

Díaz-Faes, A.A., \& Bordons, M. (2014). Acknowledgements in scientific publications: presence in Spanish science and text patterns across disciplines. Journal of the Association for Information Science and Technology, 65(9), 1834-1849. doi:10.1002/asi.23081 
This is a postprint version of:

Morillo, F.; Costas, R. \& Bordons, M. (2015). How is credit given to networking centres in their publications? A case study of the Spanish CIBER research structures. Scientometrics, 103(3), 923-938.

The final publication is available at Springer via http://dx.doi.org/10.1007/s11192-015-1564-z

García-Zorita, C., Martín-Moreno, C., Lascurain-Sánchez, M. L., \& Sanz-Casado, E. (2006). Institutional addresses in the Web of Science: the effects on scientific evaluation. Journal of Information Science, 32(4), 378-383. doi:10.1177/0165551506065813

Garner, J., Porter, A. L., Newman, N. C., \& Crowl, T. A. (2012). Assessing research network and disciplinary engagement changes induced by an NSF program. Research Evaluation, 21(2), 89-104. doi:10.1093/reseval/rvs004

Jiang, Y., Zheng, H.-T., Wang, X., Lu, B., \& Wu, K. (2011). Affiliation disambiguation for constructing semantic digital libraries. Journal of the American Society for Information Science and Technology, 62(6), 1029-1041. doi:10.1002/asi.21538

Morillo, F., Díaz-Faes, A.A., González-Albo, B., \& Moreno, L. (2014). Do networking centres perform better? An exploratory analysis in Psychiatry and Gastroenterology/Hepatology in Spain. Scientometrics, 98(2), 1401-1416. doi:10.1007/s11192-013-1183-5

Morillo, F., Santabárbara, I., \& Aparicio, J. (2013). The automatic normalisation challenge: detailed addresses identification. Scientometrics, 95(3), 953-966. doi: 10.1007/s11192-013-0965-0

Méndez Vásquez, R. I., Suñén Pinyol, E., Olivé Vázquez, G., Cervelló González, R., \& Camí, J. (2009). Caracterización bibliométrica de la produccién en Epidemiología y Salud Pública (EPI-SP) de España, 1997-2006. Barcelona. Retrieved from http://bac.fundaciorecerca.cat/ciberesp/docs/Bibliometria CIBERESP.pdf. Accessed 3 Dec 2014.

Praal, F., Kosten, J., Calero-Medina, C., \& Visser, M. S. (2013). Ranking universities: The challenge of affiliated institutes. In Translational twists and turns: science and socio-economic endeavour. Proceedings of STI 2013 Berlin. Retrieved from http://www.forschungsinfo.de/STI2013/download/STI 2013 Proceedings.pdf. Accessed 3 Dec 2014.

Rank, D., \& Williams, D. (1999). Partial benefit/cost in the evaluation of the Canadian networks of centres of excellence. Evaluation and Program Planning, 22(1), 121-129. doi:10.1016/S01497189(98)00048-2

Reijnhoudt, L., Costas, R., Noyons, E., Boerner, K., \& Scharnhorst, A. (2013). 'Seed+Expand': A validated methodology for creating high quality publication oeuvres of individual researchers. arXiv preprint arXiv:1301.5177. Accessed 3 Dec 2014.

Sirtes, D. (2013). Funding Acknowledgments for the German Research Foundation (DFG). The dirty data of the Web of Science database and how to clean it up. In Proceedings of ISSI 2013 Vienna. Retrieved from http://www.issi2013.org/Images/ISSI Proceedings Volume I.pdf. Accessed 3 Dec 2014. 
This is a postprint version of:

Morillo, F.; Costas, R. \& Bordons, M. (2015). How is credit given to networking centres in their publications? A case study of the Spanish CIBER research structures. Scientometrics, 103(3), 923-938.

The final publication is available at Springer via http://dx.doi.org/10.1007/s11192-015-1564-z

Smalheiser, N. R., \& Torvik, V. I. (2009). Author name disambiguation. Annual Review of Information Science and Technology, 43(1), 1-43. doi:10.1002/aris.2009.1440430113

Waltman, L., van Eck, N. J., van Leeuwen, T. N., Visser, M. S., \& van Raan, A. F. J. (2011). Towards a new crown indicator: Some theoretical considerations. Journal of Informetrics, 5(1), 37-47. doi:10.1016/j.joi.2010.08.001

Wang, J., \& Shapira, P. (2014). Is there a relationship between research sponsorship and publication impact ? An analysis of funding acknowledgments in nanotechnology papers. Retrieved from http://media.eurekalert.org/aaasnewsroom/MCM/FIL 000000001425/funding and research im pact-rev 2014.pdf. Accessed 11 Sep 2014 